\title{
The Design of a Novel Survey for Small Objects in the Solar System
}

C. Alcock, K. Cook, S. Marshall, C. Akerlof, W. P. Chen, I. De Pater, T. Lee, J. Lissauer, T. McKay, J. Rice and C-I. Liang

\section{August 21, 2000}




\section{DISCLAIMER}

This document was prepared as an account of work sponsored by an agency of the United States Government. Neither the United States Government nor the University of California nor any of their employees, makes any warranty, express or implied, or assumes any legal liability or responsibility for the accuracy, completeness, or usefulness of any information, apparatus, product, or process disclosed, or represents that its use would not infringe privately owned rights. Reference herein to any specific commercial product, process, or service by trade name, trademark, manufacturer, or otherwise, does not necessarily constitute or imply its endorsement, recommendation, or favoring by the United States Government or the University of California. The views and opinions of authors expressed herein do not necessarily state or reflect those of the United States Government or the University of California, and shall not be used for advertising or product endorsement purposes.

Work performed under the auspices of the U. S. Department of Energy by the University of California Lawrence Livermore National Laboratory under Contract W-7405-Eng-48.

This report has been reproduced directly from the best available copy.

Available to DOE and DOE contractors from the

Office of Scientific and Technical Information

P.O. Box 62, Oak Ridge, TN 37831

Prices available from (423) 576-8401

http://apollo.osti.gov/bridge/

Available to the public from the National Technical Information Service

U.S. Department of Commerce 5285 Port Royal Rd., Springfield, VA 22161 http://www.ntis.gov/

OR

Lawrence Livermore National Laboratory Technical Information Department's Digital Library http://www.llnl.gov/tid/Library.html 


\title{
LDRD Final Report for 97-ERI-004 \\ Exploratory Research in the Institutes
}

\section{The Design of a Novel Survey for Small Objects in the Solar System}

\author{
Principal Investigator: Charles Alcock (L-413, Extension 3-0666) \\ Co-Investigators: Kem Cook, Stuart Marshall \\ University collaborators: Carl Akerlof (University of Michigan) \\ Wen Ping Chen (National Central U., Taiwan) \\ Imke de Pater (UC Berkeley) \\ Typhoon Lee (Inst. of Astronomy, Taiwan) \\ Jack Lissauer (SUNY Stony Brook) \\ Timothy McKay (University of Michigan) \\ John Rice (UC Berkeley) \\ Graduate student: Chyng-lan Liang (UC Berkeley)
}

\begin{abstract}
We evaluated several concepts for a new survey for small objects in the Solar System. We designed a highly novel survey for comets in the outer region of the Solar System, which exploits the occultations of relatively bright stars to infer the presence of otherwise extremely faint objects. The populations and distributions of these objects are not known; the uncertainties span orders of magnitude! These objects are important scientifically as probes of the primordial solar system, and programmatically now that major investments may be made in the possible mitigation of the hazard of asteroid or comet collisions with the Earth.
\end{abstract}

\section{Scientific Motivation}

The observed frontier of the Solar System has progressed only from 10 AU when Kepler deduced his "laws" of planetary motion in the early 1600's (when only six planets were known to orbit the Sun), to $\sim 50$ AU today, with the detections of solid objects in the trans-Neptunian region (principally through the efforts of Jewitt and Luu, 1995; and Jewitt, Luu, and Chen, 1996). During these four centuries, three planets, hundreds of comets, and thousands of asteroids have been detected in orbit about the Sun, and dozens of moons as well as four ring systems have been observed to orbit planets. A current listing of trans-Neptunian objects may be found on the web (http://cfa-www. harvard.edu/cfa/ps/lists/TNOs.html and http://galileo.ifa.hawaii.edu/users/jewitt/kb.html). Progress is very slow beyond Neptune, because the objects are small (mostly small solid bodies), and the brightness in reflected sunlight declines $\sim \mathrm{r}^{-4}$. 
While no objects have been observed beyond $50 \mathrm{AU}$ (the spectacular object 1996TL66 was observed close to perihelion by Luu et al., 1997), a rich population of comets has been inferred. This population acts as a reservoir from which new comets can enter the inner Solar System, become active, and be discovered as their brightnesses increase by many orders of magnitude.

The orbits of all active comets are dynamically short-lived, in the sense that they must have been altered substantially by perturbations of planets (or, in the case of dynamically new comets, by passing stars or the galactic tidal field) in the geologically recent past. The source and/or storage regions for comets have been deduced from cometary orbits, many of which enter the planetary region on highly eccentric orbits with semi-major axes of tens of thousands of AU. Oort (1950) hypothesized a giant "cloud" of comets, tens of thousands of AU from the Sun, whose members are occasionally perturbed by passing stars into orbits that come into the planetary region. Although no bodies have been actually observed within the Oort cloud, additional data and modeling have supported and refined Oort's basic model. Dynamically new comets enter the inner Solar System from orbits with semi-major axes of order 20,000 AU as a result of perturbations by the galactic tidal field, passing stars, and occasionally passing molecular clouds (e.g., Hut and Tremaine, 1985). Encounters of the Solar System with giant molecular clouds strip away many of the comets with semi-major axes greater than 10,000 AU; thus, bodies farther from the Sun than this are unlikely to have survived over the age of the Solar System. An inner Oort cloud of icy bodies with semi-major axes of a few thousand to several thousand AU could re-supply the traditional outer Oort cloud as a result of perturbations by the same giant molecular clouds that strip the outer Oort cloud (Hills, 1981). Both the inner and outer Oort clouds could have been populated by planetesimals perturbed primarily from the Uranus/Neptune region of the protoplanetary disk during the later stages of the planet formation epoch (Duncan et al., 1987, and references therein); this model is consistent with current models of the growth of giant planets (Lissauer et al., 1995). Estimates of the total number of comet-size bodies within both the inner and outer Oort cloud range from $\sim 10^{12}$ through $\sim 10^{14}$, with values of order $10^{13}$ preferred (Weissman, 1991; Mumma et al., 1993).

The low orbital inclinations of most Jupiter family comets imply that the vast majority of them did not come from the Oort cloud, where perturbations randomize inclinations relative to the Laplacian plane of the planetary system (Duncan et al., 1988). Rather, almost all observed Jupiter family comets have spent most of their lives orbiting just beyond Neptune, within a flattened annulus known as the Kuiper Belt (Kuiper, 1951; Fernandez, 1980; and see also Edgeworth, 1949, who anticipated Kuiper's argument). A few dozen of the larger members $(\mathrm{a} \sim 100 \mathrm{~km})$ of the Kuiper Belt have now been identified (Jewitt and Luu, 1995; Jewitt, Luu, and Chen, 1996). A statistical analysis of carefully stacked HST images implies a much larger population of bodies comparable in size to large comets (10-20 km) between 30 and $45 \mathrm{AU}$ from the Sun (Cochran et al., 1995). Kuiper Belt objects are presumably formed in situ, beyond the orbits of the known major planets, and thus provide clues to the mass distribution and accretion process in this region of the 
protoplanetary disk.

Studies of the Kuiper Belt have been reviewed recently by Weissman (1995) and Stern (1996). A rich population can reasonably but indirectly be inferred. In particular, from models of the lifetimes of the Jupiter family comets, and of the dynamical lifetimes of objects in the trans-Neptunian region, a population of $\sim 10^{10}$ objects is inferred to exist in the Kuiper Belt inside $~ 50$ AU. To quote Weissman (1995): "The existence of a large number of fainter comets in the Kuiper Belt seems inescapable" (here "fainter" refers to the direct detections).

An additional feature of much of this work is the conclusion that the surface density of this population probably increases with semi-major axis out to $\sim 45 \mathrm{AU}$, after which it probably falls off $\sim \mathrm{r}^{-2}$. This implies a total population that may be more than an order of magnitude greater than that inside $50 \mathrm{AU}$; there is not even indirect evidence for or against objects in this outer part of the Kuiper Belt since such bodies would be in quite stable orbits and thus not drift into the planetary region. Bodies in the outer Kuiper Belt would be very faint and thus quite difficult to detect directly. This region is thus the least well surveyed portion of the Solar System, a place we know even less about than the Oort cloud, which is hundreds of times as far away.

This question of how far out the Kuiper Belt extends is fascinating in its own right. In addition, it is directly relevant to theories of Solar System formation, as it will provide a diagnostic of conditions in the outer reaches of the Solar System (Safronov, 1969; Stern, 1991; Lissauer, 1993). At present we do not know whether the solar nebula ended abruptly just beyond the orbit of Neptune (with the bodies detected in the Kuiper Belt thus far being the stragglers formed at the very extremities of the disk, in which case we will see very few bodies at $100 \mathrm{AU}$ ), or if the Sun's disk extended out much farther and the inner part of the Kuiper Belt is depleted relative to regions farther from the Sun as a result of perturbations by Neptune. These issues are also relevant to studies of dust disks and possible comet clouds around other stars (Smith and Terrile, 1984). A better census of the inner portion of the Kuiper Belt is also relevant to the frequency of biological extinction events, since short-period comets/extinct comets may contribute a significant fraction of the flux of Earth-crossing bodies of sufficient mass to cause such havoc.

The indirect arguments summarized above are logical and often compelling, but cry out for direct observation. Since the objects in question are so faint, an occultation approach has the advantage of "borrowing" the light from a much brighter object, a neighboring star.

The telescope system that we have designed would greatly expand our knowledge of the distribution of comet-sized bodies within the inner portion of the Kuiper disk. More fundamentally, it will be able to detect small solid objects out to $100 \mathrm{AU}$ (and even more distant larger objects). 


\section{Occultation of Stars by Comets and Asteroids}

The idea of the occultation technique is simple to describe (Bailey, 1976; Axelrod et al., 1992). One monitors the light from a sample of stars that have angular sizes smaller than the expected angular sizes of comets. An occultation is manifested by detecting the partial or total reduction in the flux from one of the stars for a brief interval. The rate of occultations is recorded over the time span of the observations. The measured rate is proportional to the number of objects, and the measured durations of the occultations give size information. The implementation of this idea is complicated by the short expected duration of an occultation event, by the large average interval between events, and by Fresnel diffraction diluting the depth of the occultation. Furthermore, the selection of stars with small angular sizes means that relatively faint stars are used; this increases the probability of false events due to shot noise in the photons. The limitations on this technique are thus set by diffraction, and by the finite sizes of the target stars.

For example, consider a comet at distance $r$ from the Earth, which is occulting a distant star. The photons have wavelength $\lambda$, $\tau$ he comet has diameter $a$. A deep occultation (i.e., a geometrical optics occultation) can occur only if a $\geq(\mathrm{r} \lambda)^{1 / 2}=(\mathrm{r} /$ $100 \mathrm{AU})^{1 / 2}(\lambda / 5000 \AA)^{1 / 2} \mathrm{~km}$. (Smaller comets do modulate the light from a background star, but by an amount $\propto \mathrm{a}^{4}$ for comets smaller than this limit.)

We must also ensure that the angular size of the target stars is smaller than that of the occulting object. The angular diameter of a $3-\mathrm{km}$ object at $100 \mathrm{AU}$ is $4 \times 10^{-10}$ radians. Typical field stars range from spectral type $A O$, radius $2 \times 10^{11} \mathrm{~cm}$, absolute visual magnitude +0.9 to $\mathrm{K} 5$ dwarfs, radius $5 \times 10^{10} \mathrm{~cm}$, absolute visual magnitude +6.8 . For stars at $m_{V}>13.5$, the $K$ dwarf will have an angular size of less than $7 \times$ $10^{-11}$ radians, and the A star will be about $5 \times 10^{-12}$ radians. Hence, our system should function with stars of this typical brightness.

The duration of the event is determined by the time the camera moves through the shadow of the comet. The Earth moves at $30 \mathrm{~km} \mathrm{~s}^{-1}$; with projection effects, the typical relative velocity will be $\sim 20 \mathrm{~km} \mathrm{~s}^{-1}$. For a 3-km comet, a strong occultation will have duration $\sim 0.2$ seconds. This is the shortest duration strong event that we anticipate being able to measure due to comet occultations. Larger comets will produce proportionately longer occultations. Note that the duration does not depend strongly on the semi-major axis of the comet's orbit; thus, the occultation gives angular size information directly, independent of any assumption regarding the albedo.

We can estimate the occultation rate due to Kuiper Belt objects as follows. Every half year, each comet "covers" a strip of sky of width (a/r) and length $(2 \mathrm{AU} / \mathrm{r})$. We estimate that the objects we seek have inclinations $\leq 12^{\circ}$; thus, in one full year, each object covers a fraction $\mathrm{f}=2(\mathrm{a} / \mathrm{r}) \times(2 \mathrm{AU} / \mathrm{r}) /\left(4 \pi \sin 12^{\circ}\right)$ of the Kuiper Belt. Hence, the number of occultations detected per year will be this fraction $\mathrm{f}$ multiplied by the number of detectable comets, times the number of monitored stars. First we consider the population of objects reported by Cochran et al. (1995), i.e., $2 \times 10^{8}$ comets with diameters of 10-20 km between 30 and $40 \mathrm{AU}$. Each of these objects will cover $10^{-10}$ of the region within $12^{\circ}$ of the ecliptic per year. The designed system 
would monitor $\sim 3,000$ stars with an $18 \%$ duty cycle, and we expect $~ 11$ events from the Cochran et al. population per year. Since these objects have a mean size of about $13 \mathrm{~km}$, the occultations would last about 0.7 seconds, which will be very easily detected by the system described below. (In fact, the telescope system described below will be able to detect 0.7 -second occultations in fainter stars, and hence the event rate due to the Cochran et al. population will be higher than the estimate given here.)

The purpose of our designed system is to detect objects much smaller than those reported by Cochran et al, or at greater distances. Cochran et al. reported that the size distribution of the objects they detected is a power law with a (differential) slope between -3 and -5 . These limiting size distributions produce 60 to 1,260 occultations per year, respectively, in the experiment we propose here. Furthermore, if this population extends beyond $45 \mathrm{AU}$, then we can expect an even higher event rate; for instance, if the surface density for these objects is proportional to $\mathrm{r}^{-2}$ out to $80 \mathrm{AU}$, then the occultation rate will be $\sim 3 \times$ the rates quoted above.

We now turn to the possible utility of follow-up observations by large telescopes. Follow-up observations will allow, on selected occasions and for bright enough objects, the direct detection of the image in visible imaging. The scientific returns from (even a small number of) detections of this kind would be great. The combination of occultation duration with apparent magnitude allows a direct estimate of the albedo of the object; this would be the first measurement of albedo for a comet presently in the trans-Neptunian region. A further return would be the possibility of obtaining orbital information from the motion of the object on the sky. Since the occultation survey alone does not provide information even on semimajor axes, this would be an extremely useful augmentation. Finally, and not to be underestimated, follow-up observations can readily and unambiguously distinguish trans-Neptunian objects from asteroids and other solid bodies which are much closer; occulting asteroids would be so bright in follow-up images that even relatively small telescopes may be used to detect them.

Follow-up observations depend on near real-time analysis of the data and the routine provision of lists of occultations to the astronomical community. This is already done by the Macho Project for microlensing events (see http://darkstar.astro.washington.edu/). The ability to process the data in near-real time will allow us to provide alerts to the community so that the occulting object may be directly detected. For an object at $40 \mathrm{AU}$ from the sun and at opposition, its apparent motion will be mostly the reflex of the Earth's motion. This will produce an apparent motion of about 70 arcsec per day to the west. A $200 \mathrm{~km}$ (diameter) object with an albedo of about 0.04 will have an apparent magnitude of about 23 mag in $\mathrm{R}$ band. This magnitude object is easily accessible with $2 \mathrm{~m}$-class ground-based telescopes. The much smaller objects that we will principally be reporting will be more difficult to detect. For instance, a $10 \mathrm{~km}$ (diameter) object would be at $\mathrm{R} \sim 29.5$; this could be detected with large telescopes, such as Keck.

Most major observatories are from 8 to 19 hours west of Taiwan and so the occulting object will have moved comfortably away from the occulted bright star. Sets of observations separated by a few hours will allow the unambiguous 
determination that any detected object near the bright source star is the occulting object. This can be done by extrapolating any apparent motion detected in the set of observations back to the known time and place of the occultation.

\section{Robotic Camera System Design}

\section{III.1 Design Criteria}

The occultation survey must have the following capabilities:

- make photometric measurements rapidly (5 Hertz);

- follow enough stars $(\sim 3,000)$;

- low false positive rate ( $\sim 0.1$ false positive per year);

- moderate cost;

- $\quad$ credible result when program complete.

Our approach to these goals is described in detail below. The rapid photometry would be achieved by trailing the images of target stars across a CCD chip at $\sim 5$ pixels per second. An adequate number of stars can be imaged onto a modern CCD camera mounted on a small $(50 \mathrm{~cm})$, wide field-of-view (f/1.9) telescope. These two considerations are not difficult to deal with.

Moderate cost can be achieved by using components that are readily available without customizing.

The low false positive rate is of great concern, and is a primary driver in the design. Our design goal is to be able to measure a rate of stellar occultations by comets of 1 per 1000 stars per year of observing time. The system should make $10^{11}$ photometric measurements per year, from which we want to derive less than one spurious occultation. The "false alarm" probability per observation must therefore be exceedingly small if the results of the experiment are to be interpreted with confidence. We require from our system a false alarm probability per measurement of $\sim 10^{-12}$, resulting in a false alarm probability per year of approximately 0.1 .

False alarms can arise from a variety of causes. A major cause is the fluctuation in the number of photons detected from a light source of constant intensity due to Poisson statistics. False alarms due to this can be controlled only by detecting a sufficiently great average number of photons from the star. This clearly has implications for the telescope aperture required and the faintness of the stars that can be utilized. Extreme atmospheric fluctuations may also pose a threat of false alarms. Additionally, false alarms can occur when a star is occulted by some terrestrial object. Birds and bats appear to be the most damaging terrestrial objects due to their potentially great numbers. (Orbital debris can be neglected due to the short time scale $\left[<10^{-3} \mathrm{~s}\right]$ of the occultation events.)

Our design controls these sources of false alarms by requiring coincident detections by three separated telescopes. These telescopes will be installed along an east-west line. The distinctive signature of a comet event is that the shadow moves from east to west at a well determined rate. There is sufficient redundancy in the 
system that false alarms due to near-field obscuration can be controlled.

The three-element design also allows us to address our last bullet. We do not believe we could credibly report on rare occultation events that were seen in only one detector. The great redundancy in the multi-telescope system greatly enhances the credibility of the reported result.

\section{III.2 Proposed System}

The proposed system consists of separated telescopes at a dark site, each with a wide-field-of-view CCD camera system pointed at the same region of the sky. This design was arrived at following a design study which determined the parameters of the elements.

Three telescopes is the minimum number needed to provide systematic control and measurement of the false positive rate (see III.3 below); we adopt this minimum number in order to minimize the overall cost of the array. With three telescopes, and the design goal of a false alarm probability per measurement of $\sim 10^{-12}$, the false alarm probability per measurement at one telescope should be no poorer than $\sim 10^{-4}$. This sets the signal to noise requirement for each telescope in the system.

The mode of operation (see II.2.d below) was chosen to achieve the high photometric measurement rate at moderate cost. The measurement rate is determined by the angle subtended by one CCD pixel on the sky, which should be 3" to ensure the desired 0.2 second measurement rate. The physical size of the pixel is determined by the availability of moderately priced camera equipment to be 14 microns. This is turn determines the focal length of the telescope and the solid angle subtended by the focal plane. Finally, the requirement that we have $>3,000$ stars on the focal plane with adequate signal to noise determines the minimum acceptable aperture of the telescope. The results of these determinations are given below.

III.2.a. The telescopes: Each automated telescope will have an aperture of $50 \mathrm{~cm}$, imaging at Cassegrain focus at $\mathrm{f} / 1.9$. The corrected field-of-view includes 3 square degrees. A telescope including the mount, optics, drives, and control system that is suitable for this project is available from Torus Instruments of Iowa City, Iowa.

III.2.b. The cameras: The imaging systems will each consist of a thermoelectrically cooled CCD camera with a $2048 \times 2048$ pixel CCD detector mounted at Cassegrain focus. Apogee Instruments of Tucson, Arizona, produces an instrument that is ideal for this purpose (Model AP10). It has 14 micron pixels, which subtend 3 arcseconds at the Cassegrain focus of the telescope described above. The entire CCD covers 3 square degrees. The data stream will be transferred to a computer via a standard PCI bus data acquisition card and written to hard disk storage.

III.2.c. The mode of operation: The observation and data processing scheme is intended to use the CCD area efficiently, resulting in simplified downstream data handling requirements. Each observation will be a multi-second exposure (5 seconds at full moon, 20 seconds at new moon, since each pixel accumulates sky 
photons for the full exposure time) with the telescope fixed (no tracking). We will track between exposures to remain in our selected fields. The CCD columns will be aligned with the Right Ascension direction so that stars will travel at the sidereal rate across the frame during the exposure. We will also have the option to track and shift the star images electronically. Each stellar image will be a "stripe" across the frame. For our proposed scale of 3 arcseconds per pixel, the exposure time per pixel will be 0.2 seconds and each "stripe" will be $\sim 100$ ( 25 at full moon) pixels in length. Observing near the ecliptic, the differential angular rate of sidereal motion between the top and bottom of our frame is negligible. The 3,000 bright stars in the frame will not significantly overlap. We will read out the CCD at $\sim 2$ million pixels per second digitized to 14 bits. At this rate, we will obtain up to 15 Gbytes of raw data per night (each pixel value will take up 2 bytes of storage). Each stellar "stripe" will represent a $\sim 20$-second time history for a star (5 seconds at full moon). For our passband of 5000-7800 Angstroms, we will achieve a limiting magnitude of approximately 15.2. Each image will contain $3000 \times 100$ photometric samples.

When a time history for a star observed with the first (master) telescope is found to contain a significant drop-out ( $>3$ sigma), the corresponding stellar time histories from the other telescopes are examined for a matching drop-out. When a match is found, the original images will be archived for more careful analysis at a later time. Matching time histories for images requires synchronization of camera shutter timing. Synchronization to UT is achieved by using local GPS receivers.

III.2.d. The alert system: The analysis steps described above will be performed in real time. At the end of each night of observing, the recorded events and the images bracketing the events will be e-mailed to the scientific participants, who will examine the image data with greater care than can be achieved in the robotic operation. All events which appear to have adequate statistical significance will be placed immediately on an "alert list." This list will be sent out to a subscription list of interested e-mail recipients, and will be placed on the web page for the project.

This sort of operation has been working well for the Macho Project, and has resulted in very significant additional scientific returns (Alcock et al., 1995).

\section{Summary}

The system described here could be used to provide a unique and powerful probe of the outer limits of the Solar System. The occultation technique has been widely applied in the investigation of structure in the Solar System; the innovative feature of this design is the use of occultations as a primary survey tool. A very large number of measurements must be made in order to yield a modest number of detections. 


\section{References}

Alcock, C. A., et al. (1995). Ap.J. 463, L67.

Alcock, C. A., et al. (1997). Ap.J., 486, 697.

Axelrod, T. S., et al. (1992). In Robotic Telescopes in the 1990s, A. V. Fillipenko, editor, ASP Conference Series, 34, 171.

Bailey, M.E. (1976) Nature, 259, 290.

Cochran, A.L., et al. (1995). Ap.J. 455, 342.

Duncan, M., T. Quinn, and S. Tremaine (1987). Astron. J. 94, 1330-1338.

Duncan, M., T. Quinn, and S. Tremaine (1988). Ap.J. Lett. 328, L69-L73.

Edgeworth, K. E. (1949). MNRAS 109, 600.

Fernandez, J. A. (1980). MNRAS 192, 481.

Geary, J. C., G. A. Luppino, R. Bredthauer, R. J. Hlivak, and L. Robinson (1991). Center for Astrophysics Preprint Series No. 3217.

Hills, J. G. (1981). A.J, 86, 1730.

Hut, P., and S. Tremaine (1985). Astron. J. 90, 1548.

Jewitt, D., and J. Luu (1995). A.J. 109, 1867.

Jewitt, D., J. Luu, and J. Chen (1996). A.J. 112, 1225.

Kuiper, G. P. (1951 in Astrophysic, p. 357 (ed. J.A. Hynek, New York: McGraw-Hill).

Lissauer, J. J. (1993). "Planet formation," Annu. Rev. Astron. Astrophys. 31, 129-174.

Lissauer, J. J., J. B. Pollack, G. W. Wetherill, and D. J. Stevenson (1995). In Neptune and

(D. Cruikshank and M. S. Matthews, Eds.), University of Arizona Press, Tucson, pp. 37-108.

Luu, J. et al. (1997). Nature 387, 573.

Mills, D. L. (October 1991). Internet time synchronization: The Network Time Protocol. IEEE Trans. Communications COM-39 10, 1482-1493.

Mumma, M. J., P. R. Weissman, and S. A. Stern (1993). In Protostars and Planets III (E. H. Levy and J. I. Lunine, Eds.), University of Arizona Press, Tucson, pp. $1177-1252$.

Oort, J. H. (1950). Bull. Astron. Inst. Neth. 11, 91.

Safronov, V. S. (1969). "Evolution of the Protoplanetary Cloud and Formation of the Earth and Planets," Moscow, Nauka Press (in Russian). English translation: NASA TTF-677, 1972.

Smith, B. A., and R. J. Terrile (1984). Science 226, 1421.

Stern, S. A. (1991). Icarus 90, 271.

Stern, S. A. (1996). A.J, 112, 1203.

Weissman, P. R. (1991). Icarus 89, 190-193.

Weissman, P. R. (1995), Ann. Rev. Astron. Astrophys. 33, 327. 